\title{
Pd nanoparticles immobilized on MIL-53(Al) as highly effective bifunctional catalysts for oxidation of liquid methanol to methyl formate
}

\author{
Jian-Fang Liu ${ }^{1}$ Jin-Cheng Mu ${ }^{1}$ Rong-Xian Qin ${ }^{1}$ Sheng-Fu $\mathrm{Ji}^{1}$
}

Received: 10 September 2018 / Published online: 18 June 2019

(c) The Author(s) 2019

\begin{abstract}
A series of Pd/MIL-53(Al) heterogeneous bifunctional catalysts with different Pd contents were prepared by an impregnation method. The prepared metal-organic frameworks MIL-53(Al) and catalysts were characterized by XRD, SEM, HRTEM, FT-IR and $\mathrm{N}_{2}$ adsorption/desorption techniques. The results showed that MIL-53(Al) was synthesized successfully, and the structure was unchanged during and after the preparation of the catalysts. The Pd nanoparticles (NPs) with an average particle size of $4.6 \mathrm{~nm}$ were uniformly dispersed on the MIL-53(Al). The catalyst exhibited good catalytic activity in the selective oxidation of liquid methanol to methyl formate. Under the conditions of $150{ }^{\circ} \mathrm{C}, 2 \mathrm{MPa} \mathrm{O}_{2}$ and solvent-free for $5 \mathrm{~h}$, the conversion of methanol could reach $60.3 \%$, and the selectivity of methyl formate was up to $62.2 \%$. In addition, the Pd/MIL-53(Al) bifunctional catalyst exhibited excellent stability and maintained high catalytic activity after five cycles.
\end{abstract}

Keywords MIL-53(Al) $\cdot$ Pd $\cdot$ Bifunctional catalyst $\cdot$ Methanol $\cdot$ Methyl formate

\section{Introduction}

The liquid-phase oxidation of alcohols over supported metal catalysts is one of the important organic conversion reactions (Besson and Gallezot 2000; Dimitratos et al. 2012) and is of great significance for basic organic synthesis and fine chemical industries (Sheldon et al. 2000). Methanol, as the simplest monohydric alcohol, can be produced from coal, natural gas, biomass and even carbon dioxide directly, so it is cheap and easily obtained. It is an important $\mathrm{C} 1$ platform molecule. The increasing maturity of synthesis technology has caused a serious excess of methanol production capacity. Many high value-added chemicals, such as formaldehyde, formic acid, methyl formate and methylal, can be obtained from methanol (Yuan et al. 2013; Shi et al. 2017; Kaichev

Edited by Xiu-Qin Zhu and Xiu-Qiu Peng

Handling editor: Runduo Zhang

Sheng-Fu Ji

jisf@mail.buct.edu.cn

1 State Key Laboratory of Chemical Resource Engineering, Beijing University of Chemical Technology, Beijing 100029, People's Republic of China et al. 2014; Ai 1982). Among them, methyl formate (MF) is known as a versatile chemical intermediate (Jenner 1995; Lee et al. 1990), and its molecule contains both ester and formyl groups. So, MF has rich reaction pathways and is often used to produce formamide, dimethylformamide, acetic acid, methyl acrylate, fumigants, pharmaceuticals and so on (Sharma et al. 2016; Nielsen et al. 2007). At present, methyl formate is mainly prepared by methanol carbonylation (Head and Tabb 1984) and methanol dehydrogenation (Sodesawa et al. 1986) in industry. The sodium methoxide catalyst used in the methanol carbonylation process is easily deactivated by hydrolysis, and its strong corrosiveness will also pollute the environment. The reaction temperature required for the methanol dehydrogenation process is high, which is not conducive to reducing energy consumption. The one-step selective oxidation of methanol to methyl formate with methanol and $\mathrm{O}_{2}$ as raw materials is not limited by thermodynamic equilibrium, and is a green, economical and efficient synthesis route.

In the past few years, many metal oxides and noble metal catalysts have been used to catalyze the oxidation of methanol to methyl formate (Liu et al. 2008; Huang et al. 2012; Zhang et al. 2014; Li et al. 2013; Merte et al. 2013). Recently, supported Pd nanoparticle catalysts have received considerable attention, due to the advantages of relatively 
low cost, high activity and resistance to poisoning, making them excellent candidates for catalysis (Zhao et al. 2013; Zhang et al. 2013). Among all the catalytic systems reported so far, Pd-based catalysts are the most efficient. They can achieve high activity and selectivity simultaneously (Yin et al. 2008; Wojcieszak et al. 2013b). Wojcieszak et al. (2014b) loaded the same content ( $2 \mathrm{wt} \%$ ) of Pd nanoparticles on $\mathrm{TiO}_{2}$ and $\mathrm{SiO}_{2}$, respectively, and studied their catalytic performance in the vapor phase oxidation of methanol to methyl formate at low temperature $\left(<100^{\circ} \mathrm{C}\right)$. Over $\mathrm{Pd} / \mathrm{SiO}_{2}$ catalyst, $88 \%$ of methanol conversion and $78 \%$ of methyl formate selectivity were obtained at $80{ }^{\circ} \mathrm{C}$. Whiting et al. (2014) used a total metal loading of $1 \mathrm{wt} \% \mathrm{Au}-\mathrm{Pd} / \mathrm{TiO}_{2}$ catalyst to catalyze the vapor phase oxidation of methanol to methyl formate. Under the conditions of $30^{\circ} \mathrm{C}$, molar ratio of $\mathrm{MeOH} / \mathrm{O}_{2} / \mathrm{He}$ of 5:2.5:92.5 and gas flow rate of $60 \mathrm{~mL} /$ min, the conversion of methanol was $25 \%$ and the selectivity of methyl formate could reach $61 \%$. However, in the process of gas-phase oxidation, the carbon loss caused by the formation of carbon oxides is a major problem. The $\mathrm{CO}$ produced in the reaction will cause poisoning and deactivation of the catalyst. Besides, as well the corrosion of equipment cannot be ignored. Compared with the continuous gas-phase reaction process, the liquid-phase batch reaction process can avoid gasification of raw materials and condensation of products, save energy and the production of toxic gases such as $\mathrm{CO}$, improving the stability and reusability of the catalyst. However, there are very few studies aiming at methanol liquid-phase oxidation to high value-added products.

Metal-organic framework materials (MOFs), as emerging porous crystalline materials composed of metal ions/clusters and organic ligands, are widely used in the heterogeneous catalysis field due to their high porosity, large specific surface area and easy functionalization (Lee et al. 2009; Corma et al. 2010; Yang et al. 2017; Yang and Yan 2016, 2017). Using MOFs to confine or stabilize metal nanoparticles can effectively prevent the migration and agglomeration of metal nanoparticles without the protection of surfactants. Meanwhile, the Lewis acidity of the MOF can interact with metal particles and achieve the purpose of bifunctional catalysis (Huang et al. 2017). For example, Pd NPs supported on MIL-101 (MIL, Materials of Institute Lavoisier) could act as an effective bifunctional catalyst for the one-step synthesis of methyl isobutyl ketone (MIBK) from acetone in the presence of $\mathrm{H}_{2}$ (Pan et al. 2010). Pd@UiO-66- $\mathrm{NH}_{2}$ (UiO, University of Oslo) was used to catalyze the tandem oxidation-acetalization of benzyl alcohol and ethylene glycol with excellent activity and selectivity ( $\mathrm{Li}$ et al. 2014). MIL$53(\mathrm{Al})\left(\mathrm{Al}(\mathrm{OH})\left[\mathrm{O}_{2} \mathrm{C}-\mathrm{C}_{6} \mathrm{H}_{4}-\mathrm{CO}_{2}\right]\right)$ is one of the most stable MOF structures, and its thermal stability can reach $500{ }^{\circ} \mathrm{C}$, so it has great potential for industrial application.

To the best of our knowledge, there are no literature reports about the catalytic oxidation of methanol over MOF supported noble metal catalysts. In this work, a simple method was developed for preparing Pd/MIL-53(Al) bifunctional catalysts by assembling Pd NPs with high activity and stability in the pores of MIL-53( $\mathrm{Al})$ for methanol liquidphase oxidation to methyl formate with molecular oxygen as oxidant. The catalysts can efficiently convert methanol to methyl formate under mild conditions. In addition, the effects of different reaction conditions on the catalytic performance and the reusability of the catalysts were also investigated. Based on the characterization of the catalysts and the reaction results, the possible reaction pathways of methanol oxidation and the role of $\mathrm{Pd}$ active species in the reaction were studied.

\section{Experimental section}

\subsection{Preparation of catalysts}

\subsubsection{Synthesis of MIL-53(Al)}

MIL-53(Al) was synthesized and purified according to the previous literature (Rallapalli et al. 2010) with a slight modification. $13 \mathrm{~g} \mathrm{Al}\left(\mathrm{NO}_{3}\right)_{3} \cdot 9 \mathrm{H}_{2} \mathrm{O}$ and $2.88 \mathrm{~g}$ 1,4-benzenedicarboxylic acid $\left(\mathrm{H}_{2} \mathrm{BDC}\right)$ were dispersed in $49 \mathrm{~mL}$ deionized water. After $30 \mathrm{~min}$ of ultrasonic mixing, the mixture was transferred to a $75 \mathrm{~mL}$ Teflon-lined steel autoclave and heated at $220^{\circ} \mathrm{C}$ for $72 \mathrm{~h}$. After completion of reaction, the autoclave was naturally cooled to room temperature, and the white powder obtained from centrifugation of the reaction liquid was washed with distilled water several times until the $\mathrm{pH}$ of the filtrate was $\sim 7$. The white solid was dried in air at $80{ }^{\circ} \mathrm{C}$ for $12 \mathrm{~h}$. Then, it was extracted with hot DMF for $12 \mathrm{~h}$ to remove unreacted terephthalic acid in the MOF channels and washed with methanol three times. Finally, the solid was dried at $80{ }^{\circ} \mathrm{C}$ under vacuum for $2 \mathrm{~h}$ to obtain fine powder MIL-53(Al).

\subsubsection{Synthesis of Pd/MIL-53(Al)}

Prior to Pd loading, the MIL-53(Al) was activated at $150{ }^{\circ} \mathrm{C}$ for $12 \mathrm{~h}$ to eliminate unreacted benzenedicarboxylic acid molecules in the cavities. Pd nanoparticles were assembled in MIL-53(Al) by liquid-phase impregnation and $\mathrm{NaBH}_{4}$ reduction. The activated MIL-53(Al) was ultrasonically dispersed in $100 \mathrm{~mL}$ ethanol and mechanically stirred in a $30^{\circ} \mathrm{C}$ water bath for $30 \mathrm{~min}$, and then, a certain volume of $\mathrm{H}_{2} \mathrm{PdCl}_{4}$ solution (containing the required mass of $\mathrm{Pd}$ ) was added dropwise into the support solution with rapid stirring continued for $12 \mathrm{~h}$. The above solution was transferred to a $0{ }^{\circ} \mathrm{C}$ ice water bath, and a fresh solution of $0.1 \mathrm{M} \mathrm{NaBH}_{4}\left(\mathrm{NaBH}_{4} / \mathrm{Pd}(\mathrm{mol} / \mathrm{mol})=5\right)$ was added dropwise. The mixture was slowly stirred and 
reduced for $2 \mathrm{~h}$. The slurry was filtered, and the resulting solid was washed thoroughly with ethanol. Then, it was dried at $60{ }^{\circ} \mathrm{C}$ for $12 \mathrm{~h}$ under vacuum. Following the above steps, Pd/MIL-53(Al) catalysts with Pd loading of 0.5, 1.0, 2.0, 3.0, 4.0, 5.0 and $6.0 \mathrm{wt} \%$ were prepared.

\subsection{Characterizations}

The catalysts were characterized by X-ray diffraction (XRD) using a D/Max $2500 \mathrm{VB}$ 2+/PC diffractometer (Rigaku, Japan) with $\mathrm{Cu} \mathrm{K} \alpha$ irradiation $(\lambda=1.5418 \AA)$ at $40 \mathrm{kV}$ and $50 \mathrm{~mA}$. Scanning electron microscope (SEM) images of samples were obtained in a TESCEN MAIA3 scanning electron microscope with an accelerating voltage of $15 \mathrm{kV}$. The transmission electron microscopy (TEM) and high-resolution electron microscopy (HRTEM) images were carried out using a JEOL (JEM 2100) transmission emission microscope operated at $100 \mathrm{kV}$ accelerating voltage. Fourier transform infrared spectroscopy (FTIR) was performed on a Bruker VERTEX 70v instrument using $\mathrm{KBr}$ pellet samples. The $\mathrm{N}_{2}$ adsorption-desorption measurement of the samples was undertaken on an ASAP $2020 \mathrm{M}$ automatic specific surface area and aperture analyzer.

\subsection{Catalytic activity evaluations}

The experiments for catalytic activity evaluation were conducted in a $100-\mathrm{mL}$ stainless autoclave equipped with gas inlet and outlet, heating and stirring apparatus. First, $5 \mathrm{~mL}$ of methanol and certain amounts of catalyst were successively added into the autoclave. Then, $\mathrm{O}_{2}$ was continually injected into the autoclave to replace the air three times. When the particular pressure was reached, the gas inlet was shut down. The autoclave was then heated to the target temperature under continuous stirring and kept for a period of reaction time. After the reaction, the autoclave was cooled down to room temperature in an ice water bath. The centrifuged reaction solution was analyzed on a gas chromatograph (Beijing East \& West Analytical Instrument, GC4000A, HJ-PLOTQ, $15 \mathrm{~m} \times 0.5 \mathrm{~mm} \times 40 \mu \mathrm{m}$ ) and analyzed quantitatively with an internal standard method to calculate the conversion rate of methanol, selectivity and yield of methyl formate. On the other hand, the gas products were gathered in collecting bags. The solid catalyst was centrifuged from the reaction solution, washed with ethanol and distilled water three times and then dried overnight under vacuum. The reusability tests were carried out as the procedures above.

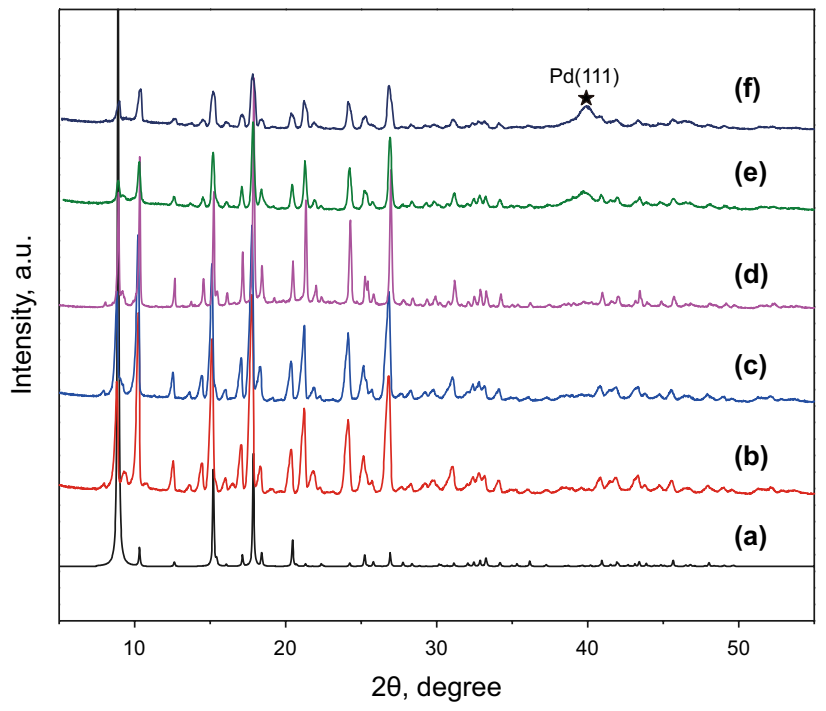

Fig. 1 XRD spectrums of as-synthesized and simulated samples. a simulated MIL-53(Al); b as-synthesized MIL-53(Al); c 0.5 wt\% Pd/ MIL-53(Al); d 1 wt\% Pd/MIL-53(Al); e 3 wt\% Pd/MIL-53(Al); and f $5 \mathrm{wt} \%$ Pd/MIL-53(Al)

\section{Results and discussion}

\subsection{Catalysts characterization}

\subsubsection{XRD}

The XRD spectra of the synthesized MIL-53(Al) and Pd/ MIL-53(Al) catalyst samples with different Pd loadings are shown in Fig. 1. From patterns (b) to (f), it can be seen that the diffraction peaks at $8.9^{\circ}, 10.3^{\circ}, 15.2^{\circ}, 17.86^{\circ}, 20.4^{\circ}$, $25.2^{\circ}$ and $26.9^{\circ}$ could correspond with the peak position of the simulated peak reported by Loiseau et al. (2004), indicating that the MIL-53( $\mathrm{Al})$ crystal structure was successfully synthesized. In the spectral lines (c) to (f), there is no obvious change in crystal structure after the loading of $\mathrm{Pd}$, indicating that MIL-53( $\mathrm{Al})$ can serve as a stable support for immobilizing Pd nanoparticles. In addition, when the Pd loading reaches $3 \mathrm{wt} \%$, the characteristic diffraction peak attributed to the $\mathrm{Pd}$ (111) crystal plane can be clearly seen at $40.1^{\circ}$ (JCPDS 46-1043) (Wang et al. 2012), and the intensity increases with the increase in the Pd loading amount, which proves successful assembling of Pd metal particles. On the other hand, when the Pd loading is less than $3 \mathrm{wt} \%$, the diffraction peak of Pd is hardly observed due to the small particle size and high dispersion.

\subsubsection{SEM and TEM}

Figure 2 shows the SEM and TEM images of synthesized MIL-53(Al) and $3 \mathrm{wt} \%$ Pd/MIL-53(Al). As can be seen from (a) and (d), MIL-53(Al) exhibits a regular nanorod structure 

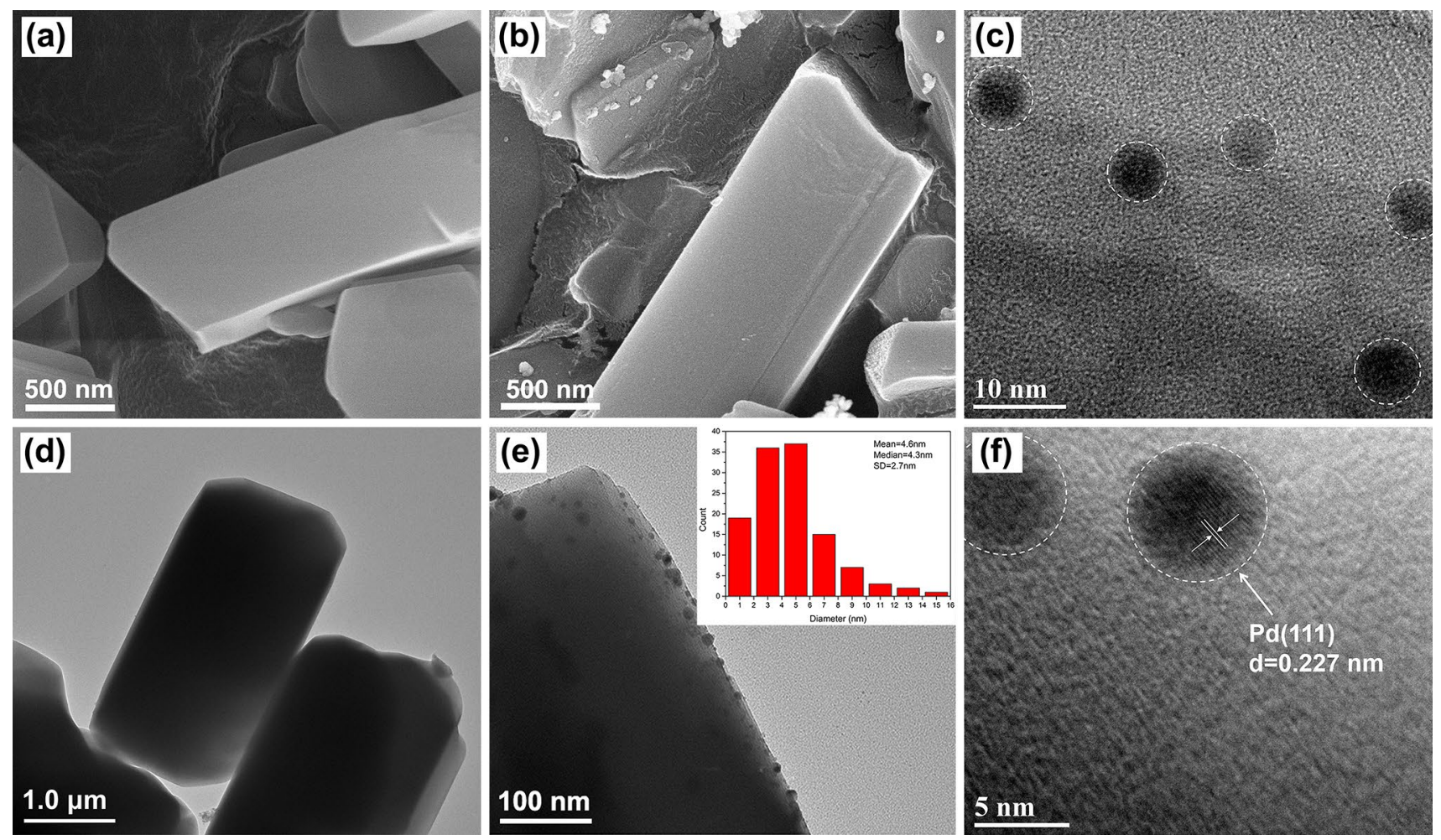

Fig. 2 SEM images of a MIL-53(Al) and $\mathbf{b} 3$ wt\% Pd/MIL-53(Al); TEM images of $\mathbf{d}$ MIL-53(Al) and e 3 wt\% Pd/MIL-53(Al) (inset is the corresponding size distribution); HRTEM images of $\mathbf{c}, \mathbf{f} 3 \mathrm{wt} \% \mathrm{Pd} / \mathrm{MIL}-53(\mathrm{Al})$, respectively

and retains its rod-like structure after Pd loading (Fig. 2b), indicating that it has good stability during the loading process. Pd NPs were highly dispersed on the outer surface of the support, and no obvious particle agglomeration was observed at the edge of the crystal. The average particle size of the Pd NPs was $4.6 \mathrm{~nm}$ after counting the particle size of more than 200 particles using Image J software (Fig. 2e). The HRTEM images of Pd NPs clearly indicate that the lattice fringe spacing is $0.23 \mathrm{~nm}$ (Fig. 2c, f), which corresponds to the (111) crystal plane of the face-centered cubic (fcc) Pd (Huang et al. 2015), and is also consistent with the XRD characterization. Pd nanoparticles with high dispersity and suitable particle size can provide great catalytic activity for the reaction (Chen et al. 2008).

\subsubsection{FT-IR}

Figure 3 shows the FT-IR spectra of MIL-53(Al) and Pd/ MIL-53(Al) with different Pd contents. MIL-53(Al) exhibits characteristic absorption peaks of carboxyl functional groups in the band of $1400-1700 \mathrm{~cm}^{-1}$ (Jiang et al. 2016). The absorption peaks appearing at 1608 and $1512 \mathrm{~cm}^{-1}$ are attributed to the asymmetric stretching vibration of $-\mathrm{COO}$, whereas the absorption peaks appearing at 1435 and $1417 \mathrm{~cm}^{-1}$ are attributed to the symmetric stretching vibration of -COO (Rallapalli et al. 2010). This demonstrates the

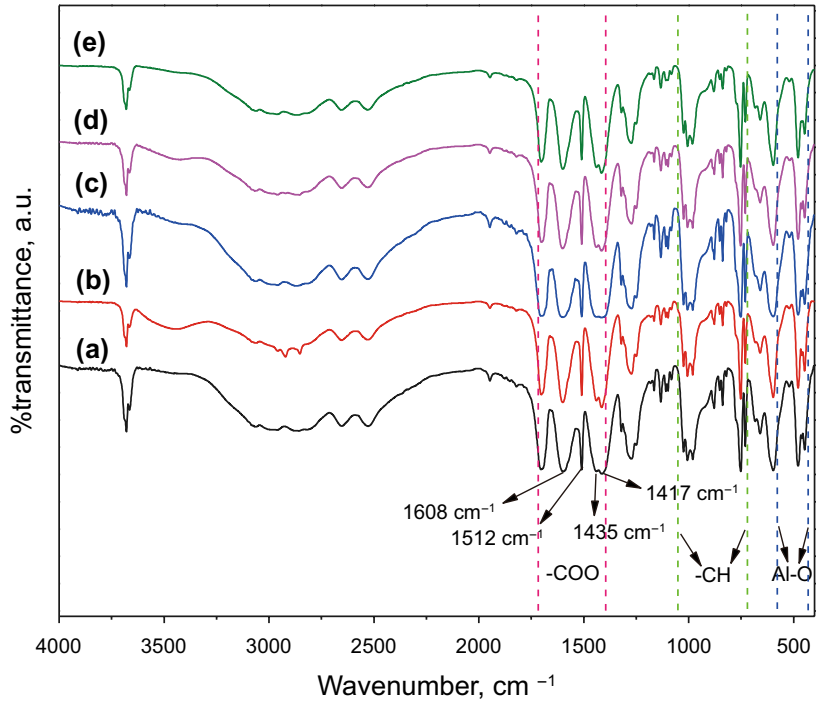

Fig. 3 FT-IR spectra of samples: a as-synthesized MIL-53(Al); b 0.5 wt \% Pd/MIL-53(Al); c 1 wt\% Pd/MIL-53(Al); d 3 wt\% Pd/MIL53(Al); and e $5 \mathrm{wt} \% \mathrm{Pd} / \mathrm{MIL}-53(\mathrm{Al})$

existence of - $\mathrm{COO}$ coordinated to the central Al. In addition, the absorption peaks between 730 and $1100 \mathrm{~cm}^{-1}$ belong to the stretching vibration of $\mathrm{C}-\mathrm{H}$, indicating the presence of the benzene ring structure in the catalyst (Rahmani and 


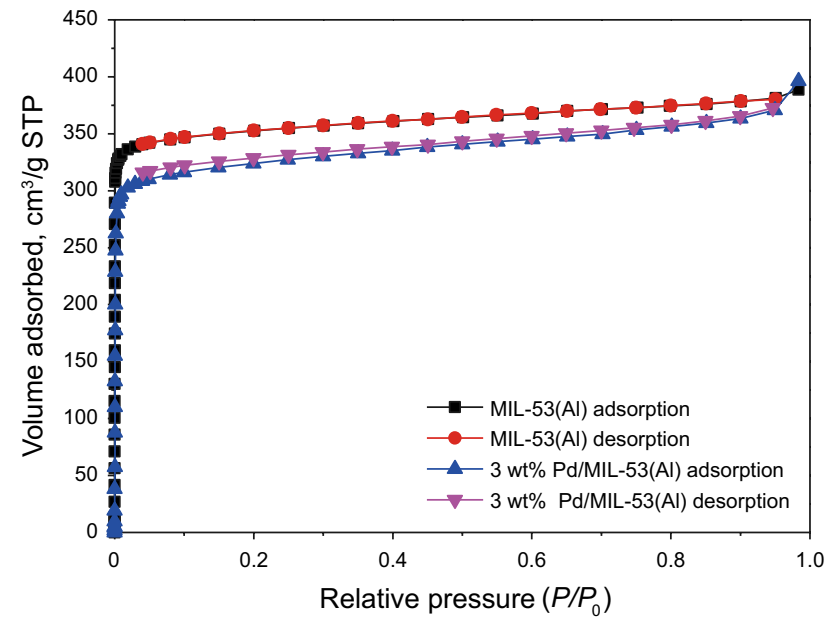

Fig. $4 \mathrm{~N}_{2}$ adsorption-desorption isotherms of the synthesized samples

Table 1 Surface area, pore volume and diameter parameter of samples

\begin{tabular}{llll}
\hline Sample & $S_{\text {BET }}, \mathrm{m}^{2} \mathrm{~g}^{-1}$ & $V, \mathrm{~cm}^{3} \mathrm{~g}^{-1}$ & $D, \mathrm{~nm}$ \\
\hline MIL-53(Al) & 1442 & 0.522 & 1.076 \\
3 wt\% Pd/MIL-53(Al) & 1302 & 0.515 & 1.076 \\
\hline
\end{tabular}

Rahmani 2017). The vibration peak in the low wavenumber range of $470-580 \mathrm{~cm}^{-1}$ is due to the presence of $\mathrm{Al}-\mathrm{O}$ in MIL-53(Al) ( $\mathrm{Li}$ et al. 2015). As the Pd loading increased from 0.5 to $5 \mathrm{wt} \%$, the peak position of the vibration peak was almost the same, and no shift occurred, indicating that the structure of MIL-53( $\mathrm{Al})$ has not been destroyed during the loading process. With the increase in loading, more Pd nanoparticles are loaded on the surface of the support, so the intensity of the characteristic peaks gradually decreases.

\subsection{4 $\mathrm{N}_{2}$ adsorption/desorption}

Figure 4 shows the $\mathrm{N}_{2}$ adsorption-desorption characteristics of MIL-53(Al) and $3 \mathrm{wt} \% \mathrm{Pd} / \mathrm{MIL}-53(\mathrm{Al})$. The BET specific surface area of the pure MIL-53(Al) support is $1442 \mathrm{~m}^{2} \mathrm{~g}^{-1}$, slightly higher than the data reported in the previous literature (Rallapalli et al. 2010), and the sharp increase in adsorption volume at low pressure $\left(P / P_{0}=10^{-6}-0.1\right)$ and the average pore size of $1.076 \mathrm{~nm}$ indicates the microporous characteristics of the material. After Pd loading, the specific surface area and pore volume of the material decreased slightly (Table 1), indicating that some of the pores of MIL53(Al) were occupied by Pd nanoparticles deposited on the surface (Gao et al. 2010; Ramos-Fernandez et al. 2012), consistent with the TEM results. Compared with traditional solid acid catalysts such as oxides and molecular sieves, the

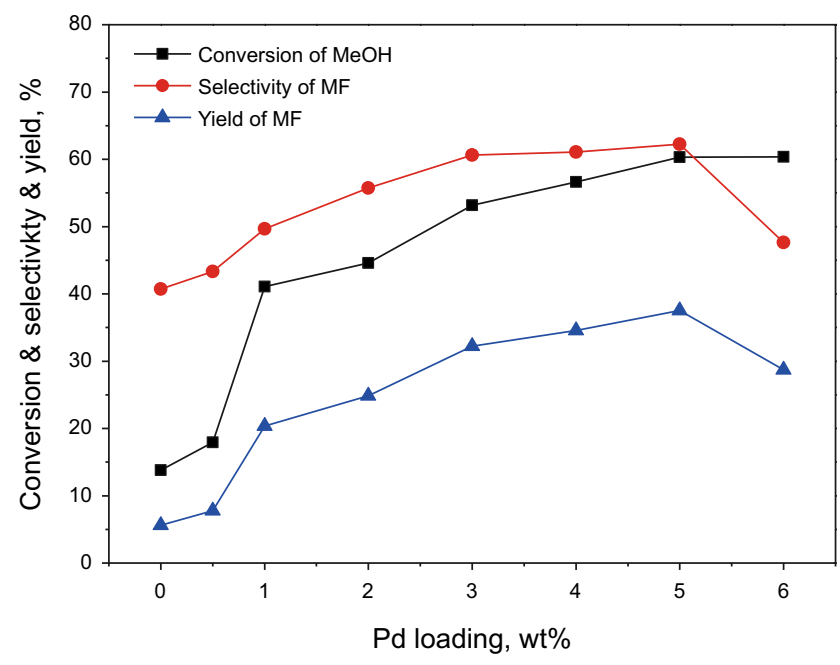

Fig. 5 Catalytic performance of Pd/MIL-53(Al) with different $\mathrm{Pd}$ loadings. Reaction conditions: $V(\mathrm{MeOH})=5 \mathrm{~mL}, P\left(\mathrm{O}_{2}\right)=2 \mathrm{MPa}$, $W(\mathrm{Pd} / \mathrm{MIL}-53(\mathrm{Al}))=50 \mathrm{mg}, T=150{ }^{\circ} \mathrm{C}, t=5 \mathrm{~h}$

larger specific surface area of MOFs can provide more active centers and contact areas, which is beneficial to the transfer and diffusion of molecules, thereby improving the yield and selectivity of specific reactions.

\subsection{Performance of liquid methanol oxidation}

Methyl formate as an important derivative of methanol is also an important intermediate in $\mathrm{C} 1$ chemistry. Methanol, as an important chemical raw material, is not only abundant, but also cheap and easily obtained. The preparation of methyl formate from methanol by one-step oxidation is a green and efficient synthetic method. However, there are few studies on liquid-phase oxidation of methanol. Based on the above characterization, the catalytic performance of $\mathrm{Pd} /$ MIL-53(Al) catalyst for liquid-phase oxidation of methanol to methyl formate was evaluated. The effects of Pd loading, catalyst dosage, reaction time, temperature, oxygen pressure for catalytic performance and reusability of catalysts were investigated.

\subsubsection{Effect of Pd loading}

Figure 5 shows the performance of Pd/MIL-53(Al) catalysts with different Pd loadings under the same conditions of $150{ }^{\circ} \mathrm{C}$ and $2 \mathrm{MPa} \mathrm{O}_{2}$. When only MIL-53(Al) was used as the catalyst without Pd loading, the activity of the reaction was extremely low. The methanol conversion rate was only $13.8 \%$, and the methyl formate yield was $5.6 \%$. With the increase in Pd loading increased from 0.5 to $5 \mathrm{wt} \%$, the methanol conversion increased from $17.9 \%$ to $60.3 \%$, and the methyl formate yield increased from $7.8 \%$ to $37.5 \%$, both significantly improved, indicating that $\mathrm{Pd}$ is the main active 


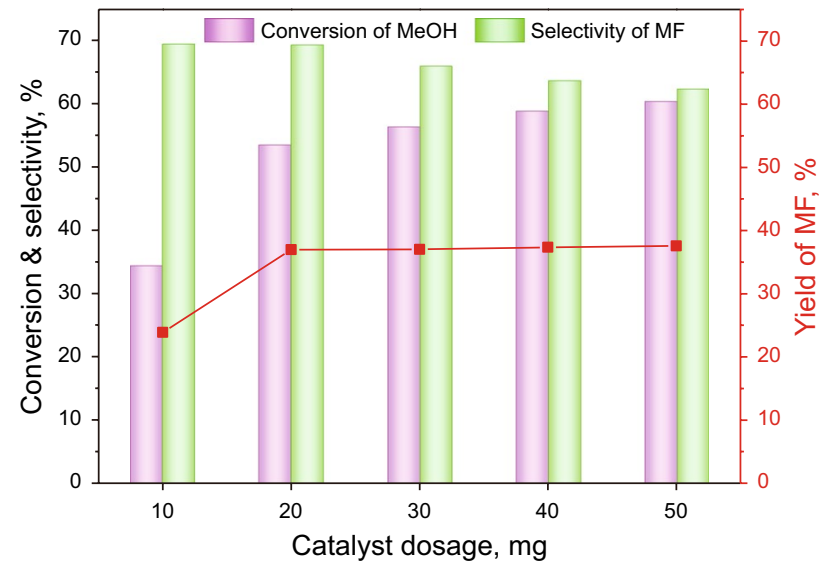

Fig. 6 Catalytic performance of Pd/MIL-53(Al) with different catalyst dosages. Reaction conditions: $V(\mathrm{MeOH})=5 \mathrm{~mL}, P\left(\mathrm{O}_{2}\right)=2 \mathrm{MPa}$, Pd loading $=5 \mathrm{wt} \%, T=150{ }^{\circ} \mathrm{C}, t=5 \mathrm{~h}$

species for the methanol oxidation reaction. As the Pd loading increases, the number of $\mathrm{Pd}$ active sites participating in the reaction also increases, and the Pd NPs can effectively enhance the ability to activate oxygen molecules (Xu et al. 2017). After continuously increasing the Pd loading to 6 $\mathrm{wt} \%$, the methanol conversion rate is basically unchanged, but the methyl formate selectivity begins to decrease. This may be due to the excessive loading of Pd and some Pd NPs being agglomerated during the preparation of the catalyst. The surface metal dispersion will decrease, and the relative content of the catalytic metal will decrease. On the other hand, the oxidability of the catalyst is dominant. The formaldehyde adsorption species on the surface of Pd (111) are oxidized to formate species and further decomposed to form $\mathrm{CO}_{2}$, which was not conducive to the formation of methyl formate (Wojcieszak et al. 2013a).

\subsubsection{Effect of the dosage of catalysts}

The effect of catalyst dosage on reaction performance is shown in Fig. 6. The reaction barely happens without any catalyst. With the increase in catalyst dosage, the number of active sites in the reaction system is gradually increased, and the conversion of methanol was also gradually increased. When the amount of catalyst increased from $10 \mathrm{mg}$ to $20 \mathrm{mg}$, the methanol conversion rate increased greatly, from $34.4 \%$ to $53.4 \%$, and the yield of methyl formate increased from $23.9 \%$ to $37.0 \%$. When the amount of catalyst continued to increase, the methanol conversion rate increased slowly, and the increase in conversion rate was not enough to match the increase in the amount of catalyst. The utilization efficiency of active sites decreased (Hou et al. 2004). When the dosage of catalyst increased from 20 to $50 \mathrm{mg}$, the selectivity for methyl formate decreased gradually, indicating that excessive catalyst would reduce the degree of dispersion of active

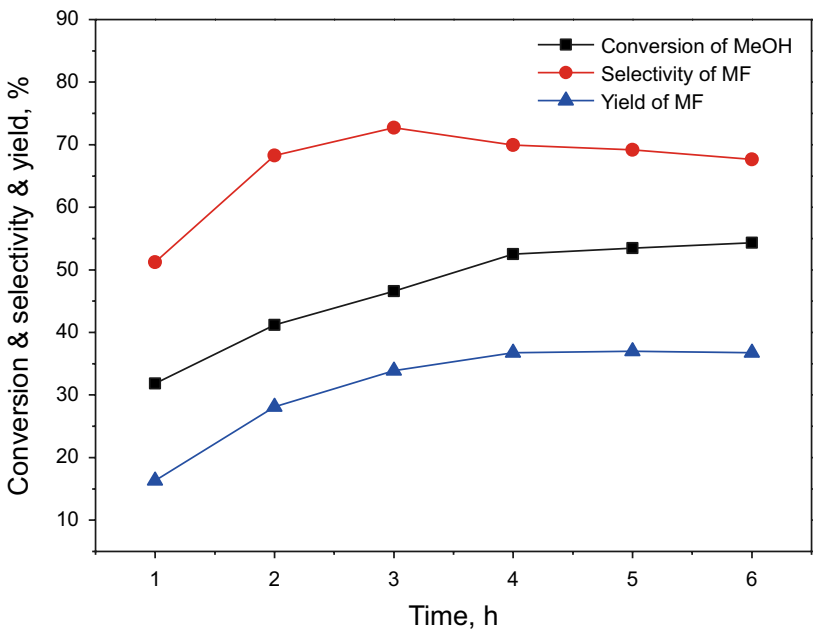

Fig. 7 Catalytic performance of $\mathrm{Pd} / \mathrm{MIL}-53(\mathrm{Al})$ at different times. Reaction conditions: $V(\mathrm{MeOH})=5 \mathrm{~mL}, P\left(\mathrm{O}_{2}\right)=2 \mathrm{MPa}$, Pd loading $=5 \mathrm{wt} \%, W(\mathrm{Pd} / \mathrm{MIL}-53(\mathrm{Al}))=20 \mathrm{mg}, T=150^{\circ} \mathrm{C}$

sites. Some active sites were covered, so the selectivity of products was affected (Sun et al. 2005). But the yield did not change significantly and reached a stable state. Therefore, considering the consumption of catalyst and the yield of the target product methyl formate, $20 \mathrm{mg}$ was chosen as the optimum catalyst dosage.

\subsubsection{Effect of reaction time}

Figure 7 shows the relationship between reaction time and catalytic performance. At the beginning of the reaction, the methanol conversion and methyl formate yield are gradually increased with the extension of reaction time. When the reaction time reached $4 \mathrm{~h}$, the growth rate of the conversion began to slow down. After $5 \mathrm{~h}$, the methanol conversion and the methyl formate yield both remained constant. The methanol conversion rate reached $52.5 \%$, and the methyl formate yield was $36.7 \%$. The reaction reached equilibrium. Up to $3 \mathrm{~h}$, the methyl formate selectivity increased from $51.2 \%$ to $72.7 \%$ as the reaction progressed. Then, as the reaction time continued to increase, the methyl formate selectivity began to slowly decrease to a stable level, which may be due to the aggregation of highly dispersed metal nanoparticles to reduce surface energy, and the catalytic performance is decreased (Kesavan et al. 2011). On the other hand, as the amount of methanol conversion increases, the collision probability between the formed formic acid intermediate and the surrounding methanol molecules decreases, so that the selectivity of esterification to form methyl formate decreases. The performance study on the reaction time shows that the catalyst has good stability in the liquid-phase methanol oxidation reaction. 


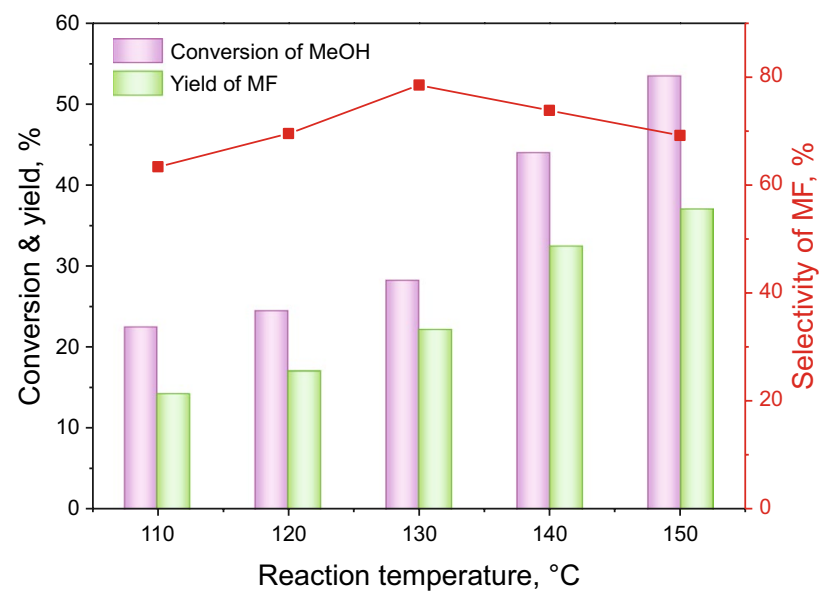

Fig. 8 Catalytic performance of Pd/MIL-53(Al) at different reaction temperatures. Reaction conditions: $V(\mathrm{MeOH})=5 \mathrm{~mL}, P\left(\mathrm{O}_{2}\right)=2 \mathrm{MPa}$, $\mathrm{Pd}$ loading $=5 \mathrm{wt} \%, W(\mathrm{Pd} / \mathrm{MIL}-53(\mathrm{Al}))=20 \mathrm{mg}, t=5 \mathrm{~h}$

\subsubsection{Effect of temperature}

Figure 8 exhibits the change in catalytic performance at different reaction temperatures. It can be seen from the figure that the temperature has a great influence on the activity of the catalyst. At less than $130{ }^{\circ} \mathrm{C}$, the methanol conversion rate hardly changes significantly (from $22.4 \%$ to $28.2 \%$ ). When the reaction temperature rises from 130 to $150{ }^{\circ} \mathrm{C}$, the methanol conversion increased rapidly to $53.4 \%$, while the selectivity of methyl formate reached the highest selectivity of $78.6 \%$ at $130{ }^{\circ} \mathrm{C}$. Then, with the further increase in temperature, the selectivity of methyl formate began to decrease gradually. Although the elevated temperature can improve the selectivity of methyl formate to a certain extent, the reaction temperature should not be too high. Because the activity of the oxygen species on the surface of the Pd catalyst is high, the adsorption capacity to the formaldehyde intermediate is also strong (Chen et al. 2004), and the methanol is easily deeply oxidized to form carbon dioxide and other by-products (Dimitratos et al. 2005), reducing the selectivity of the reaction. Considering the formation mechanism of methyl formate, methanol oxidation first generates the intermediate product formaldehyde, and it is beneficial to this reaction to raise the temperature. The dehydrogenation of methoxymethanol intermediates formed by the condensation of the adsorbed methoxides with $\mathrm{HCHO}$ (Lichtenberger et al. 2007) and the esterification of formic acid formed by further oxidation of formaldehyde and methanol to methyl formate (Wojcieszak et al. 2014a) both require increasing the number of acid sites of the catalyst to match the oxidation reaction and the condensation reaction.

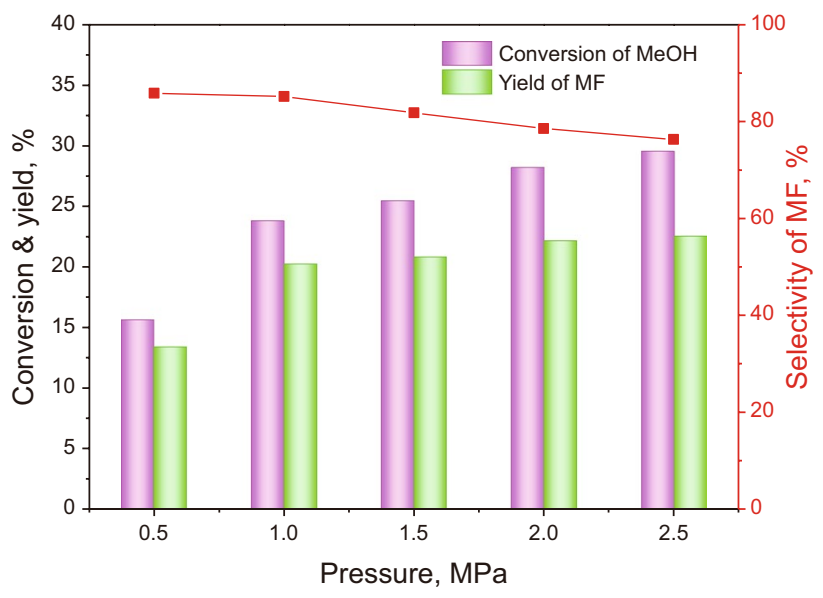

Fig. 9 Catalytic performance of Pd/MIL-53(Al) at different oxygen pressures. Reaction conditions: $V(\mathrm{MeOH})=5 \mathrm{~mL}, \mathrm{Pd}$ loading $=5$ $\mathrm{wt} \%, W(\mathrm{Pd} / \mathrm{MIL}-53(\mathrm{Al}))=20 \mathrm{mg}, T=130{ }^{\circ} \mathrm{C}, t=5 \mathrm{~h}$

\subsubsection{Effect of oxygen pressure}

The effect of the initial oxygen pressure from 0.5 to $2.5 \mathrm{MPa}$ on the reaction performance is demonstrated in Fig. 9. It can be seen from the figure that as the initial oxygen pressure increases from 0.5 to $1 \mathrm{MPa}$, the methanol conversion increases significantly from $15.6 \%$ to $23.8 \%$. When the oxygen pressure is continuously increased to $2.5 \mathrm{MPa}$, the rate of methanol conversion increases gradually and the methyl formate selectivity decreased slightly from $85.8 \%$ to $76.3 \%$ with increasing oxygen pressure. This is because as the initial oxygen pressure increases, the adsorption activation of oxygen on the surface of $\mathrm{Pd}$ is enhanced, providing more active oxygen atoms (Mate et al. 2013), which are combined with $\mathrm{H}$ adsorbed on the surface of $\mathrm{Pd}$ from $\mathrm{O}-\mathrm{H}$ cleavage in methanol and $\beta-\mathrm{H}$ elimination to form water, thus releasing the active site of Pd (Mori et al. 2004) and promoting the oxidation of $\mathrm{MeOH}$. However, if the oxygen pressure is further increased, the adsorption of oxygen molecules on the surface of Pd reaches saturation, and the rate of adsorption and dissociation is balanced (Hou et al. 2008), so the conversion of methanol is not significantly changed. At the same time, excess oxygen will continue to oxidize the $\mathrm{CO}$ formed by the decarbonylation of formaldehyde to $\mathrm{CO}_{2}$, which is so-called over oxidation (Kluytmans et al. 2000), reducing the selectivity of the target product methyl formate. Under the same operating conditions, using $\mathrm{N}_{2}$ instead of $\mathrm{O}_{2}$ to replace the air in the reactor for experiments, there is almost no conversion of methanol, indicating that $\mathrm{O}_{2}$ as the sole oxidant plays an important role in removing the adsorbed species on the catalytic active sites and renewing the active sites for the next catalytic cycle (Mallat and Baiker 2004).

Based on the above studies, a possible reaction mechanism was proposed for the selective oxidation of methanol 


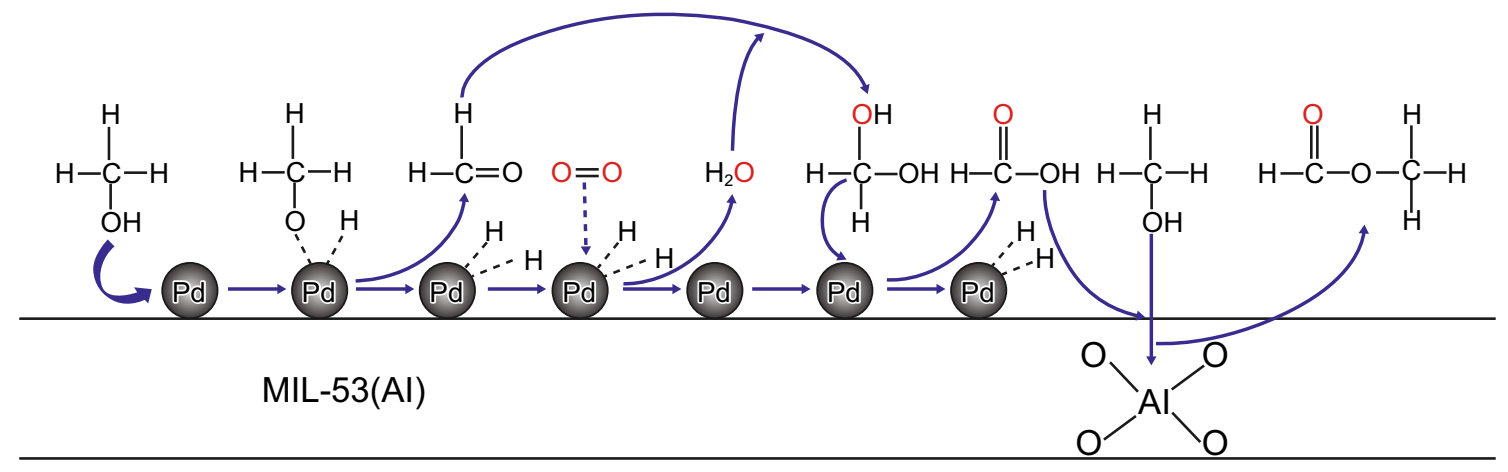

Fig. 10 Reaction mechanism of methanol selective oxidation to methyl formate on Pd/MIL-53(Al)

to methyl formate on Pd/MIL-53(Al) catalyst as shown in Fig. 10. The methanol molecules are first adsorbed on the (111) crystal plane of the Pd nanoparticles (Ferri et al. 2006), and the $\mathrm{O}-\mathrm{H}$ bond in methanol weakens, formation of $\mathrm{Pd}-\mathrm{O}$ bonds prevails, and chemisorbed methoxy species $\mathrm{CH}_{3} \mathrm{O}$ are formed (Liu and Corma 2018). Then, the adsorbed methoxide species are transformed to formaldehyde by $\beta-\mathrm{H}$ elimination, which has a higher activation energy and is the rate-determining step for the whole reaction (Guo et al. 2014). Next, the $\mathrm{O}_{2}$ molecules are adsorbed and activated on the surface of the Pd nanoparticles to generate reactive oxygen atoms [O*] (Keresszegi et al. 2002), which react with the formed metal hydride and oxidize the $\mathrm{H}$ atoms thereon to release the active sites on the metal surface and simultaneously generate $\mathrm{H}_{2} \mathrm{O}$ as the by-product (Abad et al. 2008). However, the reaction of directly inserting oxygen atoms into formaldehyde to form formic acid is usually very slow (Mallat and Baiker 1994), so it is speculated that the formation of formic acid is obtained by hydration of formaldehyde with the formed $\mathrm{H}_{2} \mathrm{O}$ molecules to form methyl glycol and then removing two hydrogen atoms on Pd (Keresszegi et al. 2005). Finally, the formic acid formed is esterified with the nearby adsorbed methanol molecules on the Lewis acid sites of the supported MIL-53(Al) and then desorbs from the surface of the support to form methyl formate.

\subsubsection{Reusability of catalysts}

In order to evaluate the stability of the catalyst, the reusability of Pd/MIL-53(Al) catalyst was tested under the same experimental conditions. As shown in Fig. 11, the catalyst showed great stability. After five times repeated use, the methanol conversion decreased from $60.3 \%$ to $57.6 \%$, and the activity of the catalyst did not change significantly. From Fig. 12, it can be seen that the metal nanoparticles in the reused catalyst remain highly dispersed without large particle agglomeration. ICP tests were carried out on the reused catalyst and reaction solution. The Pd content in Pd/MIL-53(Al) catalyst was still higher than $4.9 \mathrm{wt} \%$, and

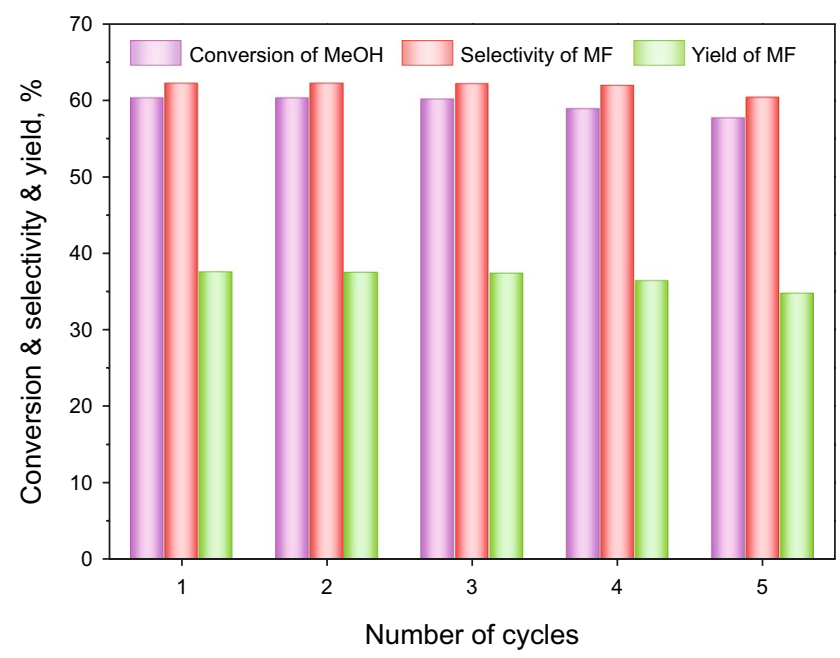

Fig. 11 Reusability of Pd/MIL-53(Al) catalyst. Reaction conditions: $V(\mathrm{MeOH})=5 \mathrm{~mL}, P\left(\mathrm{O}_{2}\right)=2 \mathrm{MPa}$, Pd loading $=5 \mathrm{wt} \%, W(\mathrm{Pd} / \mathrm{MIL}-$ $53(\mathrm{Al}))=50 \mathrm{mg}, T=150^{\circ} \mathrm{C}, t=5 \mathrm{~h}$

no Pd species was detected in the reaction solution, which also explains the reason why the catalytic activity of $\mathrm{Pd} /$ MIL-53(Al) catalyst did not decrease significantly. It shows that Pd/MIL-53(Al) catalysts have excellent stability in the liquid-phase oxidation of methanol, and the whole reaction is heterogeneous rather than homogeneous.

\section{Conclusions}

In summary, Pd nanoparticles immobilized on MIL-53(Al) were synthesized as a novel bifunctional catalyst for highly effective liquid methanol selective oxidation to methyl formate, and the structure and performance of catalysts were investigated in detail. Particularly, under the solvent-free conditions of $150{ }^{\circ} \mathrm{C}$ and $2 \mathrm{MPa}_{2}$ for $5 \mathrm{~h}$, the methanol conversion catalyzed by $5 \mathrm{wt} \%$ Pd/MIL-53(Al) could reach $60.3 \%$, and the selectivity of methyl formate was up to $62.2 \%$. The high catalytic activity of the catalyst was 

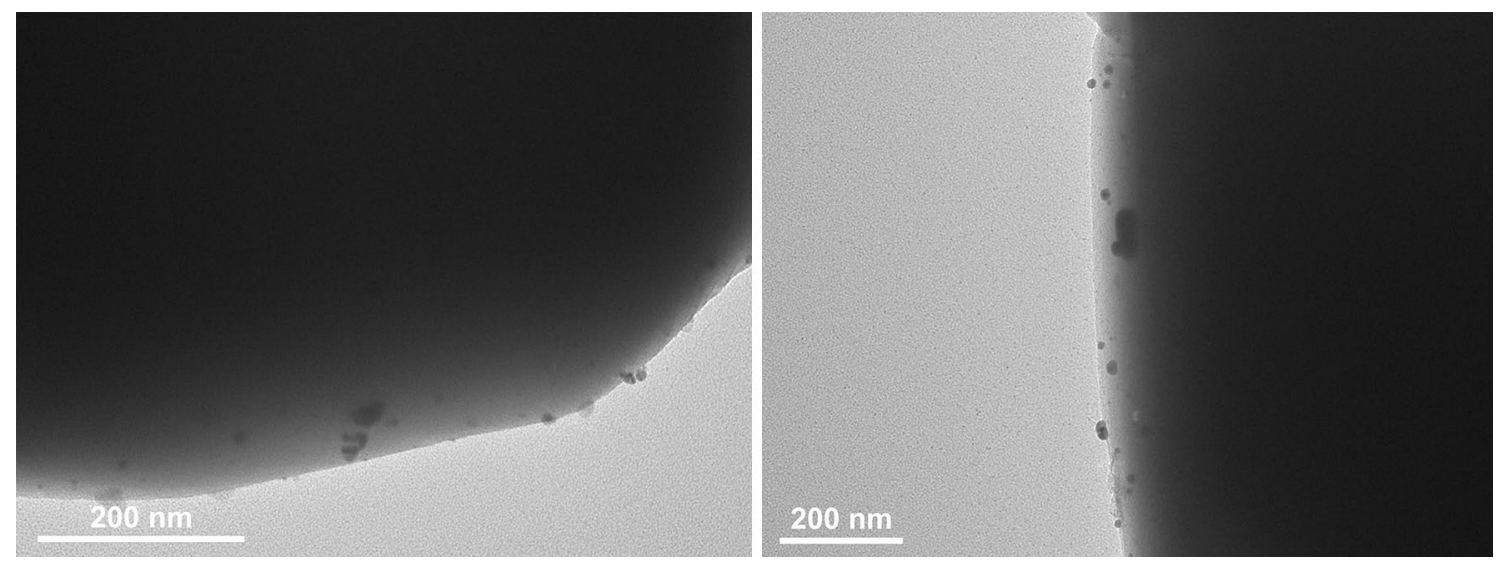

Fig. 12 TEM images of $5 \mathrm{wt} \% \mathrm{Pd} / \mathrm{MIL}-53(\mathrm{Al})$ catalyst after five reaction cycles

attributed to the unique confinement effect of the porous structure within the MOF and the highly dispersed Pd nanoparticles with an average size of $4.6 \mathrm{~nm}$. In contrast to chemically and thermodynamically unstable MOFs, $\mathrm{Pd} /$ MIL-53(Al) was readily recycled without degradation of its structure and catalytic activity. In brief, Pd/MIL-53(Al) catalysts might pave the way to one-step oxidation of liquid methanol to methyl formate, which possesses potential application prospects.

Acknowledgements Financial support received from the National Natural Science Foundation of China (Grant Nos. 21573015, 21872004) is gratefully acknowledged.

Open Access This article is distributed under the terms of the Creative Commons Attribution 4.0 International License (http://creativeco mmons.org/licenses/by/4.0/), which permits unrestricted use, distribution, and reproduction in any medium, provided you give appropriate credit to the original author(s) and the source, provide a link to the Creative Commons license, and indicate if changes were made.

\section{References}

Abad A, Corma A, García H. Catalyst parameters determining activity and selectivity of supported gold nanoparticles for the aerobic oxidation of alcohols: the molecular reaction mechanism. Chem Eur J. 2008;14(1):212-22. https://doi.org/10.1002/chem.200701263.

Ai M. The production of methyl formate by the vapor-phase oxidation of methanol. J Catal. 1982;77(1):279-88. https://doi. org/10.1016/0021-9517(82)90168-3.

Besson M, Gallezot P. Selective oxidation of alcohols and aldehydes on metal catalysts. Catal Today. 2000;57(1):127-41. https://doi. org/10.1016/S0920-5861(99)00315-6.

Chen Z-X, Neyman KM, Lim KH, Rösch N. $\mathrm{CH}_{3} \mathrm{O}$ decomposition on $\mathrm{PdZn}(111), \operatorname{Pd}(111)$, and $\mathrm{Cu}(111)$. A theoretical study. Langmuir. 2004;20(19):8068-77. https://doi.org/10.1021/la049377z.

Chen J, Zhang Q, Wang Y, Wan H. Size-dependent catalytic activity of supported palladium nanoparticles for aerobic oxidation of alcohols. Adv Synth Catal. 2008;350(3):453-64. https://doi. org/10.1002/adsc.200700350.

Corma A, García H, Llabrés i Xamena F. Engineering metal organic frameworks for heterogeneous catalysis. Chem Rev. 2010;110(8):4606-55. https://doi.org/10.1021/cr9003924.

Dimitratos N, Porta F, Prati L. Au, Pd (mono and bimetallic) catalysts supported on graphite using the immobilisation method: Synthesis and catalytic testing for liquid phase oxidation of glycerol. Appl Catal A. 2005;291(1-2):210-4. https://doi. org/10.1016/j.apcata.2005.01.044.

Dimitratos N, Lopez-Sanchez JA, Hutchings GJ. Selective liquid phase oxidation with supported metal nanoparticles. Chem Sci. 2012;3(1):20-44. https://doi.org/10.1039/C1SC00524C.

Ferri D, Mondelli C, Krumeich F, Baiker A. Discrimination of active palladium sites in catalytic liquid-phase oxidation of benzyl alcohol. J Phys Chem B. 2006;110(46):22982-6. https://doi. org/10.1021/jp065779z.

Gao S, Zhao N, Shu M, Che S. Palladium nanoparticles supported on MOF-5: a highly active catalyst for a ligand-and copperfree Sonogashira coupling reaction. Appl Catal A. 2010;388(12):196-201. https://doi.org/10.1016/j.apcata.2010.08.045.

Guo Z, Liu B, Zhang Q, Deng W, Wang Y, Yang Y. Recent advances in heterogeneous selective oxidation catalysis for sustainable chemistry. Chem Soc Rev. 2014;43(10):3480-524. https://doi. org/10.1039/C3CS60282F.

Head RA, Tabb MI. Platinum-catalysed carbonylation of methanol to methyl formate. J Mol Catal. 1984;26(1):149-58. https://doi. org/10.1016/0304-5102(84)85029-4.

Hou Y, Wang Y, He F, Mi W, Li Z, Mi Z, et al. Effects of lanthanum addition on $\mathrm{Ni}-\mathrm{B} / \gamma-\mathrm{Al}_{2} \mathrm{O}_{3}$ amorphous alloy catalysts used in anthraquinone hydrogenation. Appl Catal A. 2004;259(1):3540. https://doi.org/10.1016/j.apcata.2003.09.006.

Hou W, Dehm NA, Scott RW. Alcohol oxidations in aqueous solutions using $\mathrm{Au}, \mathrm{Pd}$, and bimetallic AuPd nanoparticle catalysts. J Catal. 2008;253(1):22-7. https://doi.org/10.1016/j. jcat.2007.10.025.

Huang H, Li W, Liu H. Effect of treatment temperature on structures and properties of zirconia-supported ruthenium oxide catalysts for selective oxidation of methanol to methyl formate. Catal Today. 2012;183(1):58-64. https://doi.org/10.1016/j.cattod.2011.05.021.

Huang Y-B, Shen M, Wang X, Shi P-C, Li H, Cao R. Hierarchically micro-and mesoporous metal-organic framework-supported alloy nanocrystals as bifunctional catalysts: toward cooperative 
catalysis. J Catal. 2015;330:452-7. https://doi.org/10.1016/j. jcat.2015.07.029.

Huang Y-B, Liang J, Wang X-S, Cao R. Multifunctional metalorganic framework catalysts: synergistic catalysis and tandem reactions. Chem Soc Rev. 2017;46(1):126-57. https://doi. org/10.1039/C6CS00250A.

Jenner G. Homogeneous catalytic reactions involving methyl formate. Appl Catal A. 1995;121(1):25-44. https://doi. org/10.1016/0926-860X(95)85008-2.

Jiang S, Yan J, Habimana F, Ji S. Preparation of magnetically recyclable MIL-53(Al)@SiO2@Fe3O4 catalysts and their catalytic performance for Friedel-Crafts acylation reaction. Catal Today. 2016;264:83-90. https://doi.org/10.1016/j.cattod.2015.10.003.

Kaichev VV, Popova GY, Chesalov YA, Saraev A, Zemlyanov D, Beloshapkin S, et al. Selective oxidation of methanol to form dimethoxymethane and methyl formate over a monolayer $\mathrm{V}_{2} \mathrm{O}_{5} /$ $\mathrm{TiO}_{2}$ catalyst. J Catal. 2014;311:59-70. https://doi.org/10.1016/j. jcat.2013.10.026.

Keresszegi C, Bürgi T, Mallat T, Baiker A. On the role of oxygen in the liquid-phase aerobic oxidation of alcohols on palladium. J Catal. 2002;211(1):244-51. https://doi.org/10.1006/jcat.2002.3723.

Keresszegi C, Ferri D, Mallat T, Baiker A. Unraveling the surface reactions during liquid-phase oxidation of benzyl alcohol on $\mathrm{Pd} / \mathrm{Al}_{2} \mathrm{O}_{3}$ : an in situ ATR-IR study. J Phys Chem B. 2005;109(2):958-67. https://doi.org/10.1021/jp0459864.

Kesavan L, Tiruvalam R, Ab Rahim MH, bin Saiman MI, Enache DI, Jenkins RL, et al. Solvent-free oxidation of primary carbon-hydrogen bonds in toluene using $\mathrm{Au}-\mathrm{Pd}$ alloy nanoparticles. Science. 2011;331(6014):195-9. https://doi.org/10.1126/science.1198458.

Kluytmans J, Markusse A, Kuster B, Marin G, Schouten J. Engineering aspects of the aqueous noble metal catalysed alcohol oxidation. Catal Today. 2000;57(1-2):143-55. https://doi.org/10.1016/S0920 -5861(99)00316-8.

Lee JS, Kim J, Kim Y. Methyl formate as a new building block in C1 chemistry. Appl Catal. 1990;57(1):1-30. https://doi.org/10.1016/ S0166-9834(00)80720-4.

Lee J, Farha OK, Roberts J, Scheidt KA, Nguyen ST, Hupp JT. Metal-organic framework materials as catalysts. Chem Soc Rev. 2009;38(5):1450-9. https://doi.org/10.1039/B807080F.

Li Z, Xu J, Gu X, Wang K, Wang W, Zhang X, et al. Selective gasphase oxidation of alcohols over nanoporous silver. ChemCatChem. 2013;5(7):1705-8. https://doi.org/10.1002/cctc.20120 0862.

Li X, Guo Z, Xiao C, Goh TW, Tesfagaber D, Huang W. Tandem catalysis by palladium nanoclusters encapsulated in metalorganic frameworks. ACS Catal. 2014;4(10):3490-7. https://doi. org/10.1021/cs5006635

Li C, Xiong Z, Zhang J, Wu C. The strengthening role of the amino group in metal-organic framework MIL-53(Al) for methylene blue and malachite green dye adsorption. J Chem Eng Data. 2015;60(11):3414-22. https://doi.org/10.1021/acs.jced.5b00692.

Lichtenberger J, Lee D, Iglesia E. Catalytic oxidation of methanol on Pd metal and oxide clusters at near-ambient temperatures. Phys Chem Chem Phys. 2007;9(35):4902-6. https://doi.org/10.1039/ B707465D

Liu L, Corma A. Metal catalysts for heterogeneous catalysis: from single atoms to nanoclusters and nanoparticles. Chem Rev. 2018;118(10):4981-5079. https://doi.org/10.1021/acs.chemr ev.7b00776.

Liu J, Zhan E, Cai W, Li J, Shen W. Methanol selective oxidation to methyl formate over $\mathrm{ReO}_{\mathrm{x}} / \mathrm{CeO}_{2}$ catalysts. Catal Lett. 2008;120(34):274-80. https://doi.org/10.1007/s10562-007-9280-9.

Loiseau T, Serre C, Huguenard C, Fink G, Taulelle F, Henry M, et al. A rationale for the large breathing of the porous aluminum terephthalate (MIL-53) upon hydration. Chem Eur J. 2004;10(6):137382. https://doi.org/10.1002/chem.200305413.
Mallat T, Baiker A. Oxidation of alcohols with molecular oxygen on platinum metal catalysts in aqueous solutions. Catal Today. 1994;19(2):247-83. https://doi.org/10.1016/0920-5861(94)80187 $-8$.

Mallat T, Baiker A. Oxidation of alcohols with molecular oxygen on solid catalysts. Chem Rev. 2004;104(6):3037-58. https://doi. org/10.1021/cr0200116.

Mate VR, Shirai M, Rode CV. Heterogeneous $\mathrm{Co}_{3} \mathrm{O}_{4}$ catalyst for selective oxidation of aqueous veratryl alcohol using molecular oxygen. Catal Commun. 2013;33:66-9. https://doi.org/10.1016/j.catco m.2012.12.015.

Merte LR, Ahmadi M, Behafarid F, Ono LK, Lira E, Matos J, et al. Correlating catalytic methanol oxidation with the structure and oxidation state of size-selected Pt nanoparticles. ACS Catal. 2013;3(7):1460-8. https://doi.org/10.1021/cs400234h.

Mori K, Hara T, Mizugaki T, Ebitani K, Kaneda K. Hydroxyapatitesupported palladium nanoclusters: a highly active heterogeneous catalyst for selective oxidation of alcohols by use of molecular oxygen. J Am Chem Soc. 2004;126(34):10657-66. https://doi. org/10.1021/ja0488683.

Nielsen IS, Taarning E, Egeblad K, Madsen R, Christensen CH. Direct aerobic oxidation of primary alcohols to methyl esters catalyzed by a heterogeneous gold catalyst. Catal Lett. 2007;116(1-2):3540. https://doi.org/10.1007/s10562-007-9086-9.

Pan Y, Yuan B, Li Y, He D. Multifunctional catalysis by Pd@MIL-101: one-step synthesis of methyl isobutyl ketone over palladium nanoparticles deposited on a metal-organic framework. Chem Commun. 2010;46(13):2280-2. https://doi.org/10.1039/B922061E.

Rahmani E, Rahmani M. Al-based MIL-53 metal organic framework (MOF) as the new catalyst for Friedel-Crafts alkylation of benzene. Ind Eng Chem Res. 2017;57(1):169-78. https://doi. org/10.1021/acs.iecr.7b04206.

Rallapalli P, Patil D, Prasanth K, Somani RS, Jasra R, Bajaj H. An alternative activation method for the enhancement of methane storage capacity of nanoporous aluminium terephthalate, MIL-53(Al). J Porous Mater. 2010;17(5):523-8. https://doi. org/10.1007/s10934-009-9320-5.

Ramos-Fernandez EV, Pieters C, van der Linden B, Juan-Alcañiz J, Serra-Crespo P, Verhoeven M, et al. Highly dispersed platinum in metal organic framework $\mathrm{NH}_{2}$-MIL-101(Al) containing phosphotungstic acid-characterization and catalytic performance. J Catal. 2012;289:42-52. https://doi.org/10.1016/j.jcat.2012.01.013.

Sharma AS, Kaur H, Shah D. Selective oxidation of alcohols by supported gold nanoparticles: recent advances. RSC Adv. 2016;6(34):28688-727. https://doi.org/10.1039/C5RA25646A.

Sheldon RA, Arends I, Dijksman A. New developments in catalytic alcohol oxidations for fine chemicals synthesis. Catal Today. 2000;57(1-2):157-66. https://doi.org/10.1016/S0920 -5861(99)00317-X.

Shi D, Liu J, Ji S. Preparation of $\mathrm{Au} / \mathrm{TiO}_{2}$ catalyst and the performance of liquid methanol catalytic oxidation to formic acid. Ind Eng Chem Res. 2017;56(39):11028-33. https://doi.org/10.1021/acs. iecr.7b02506.

Sodesawa T, Nagacho M, Onodera A, Nozaki F. Dehydrogenation of methanol to methyl formate over $\mathrm{Cu} / \mathrm{SiO}_{2}$ catalysts prepared by ion exchange method. J Catal. 1986;102(2):460-3. https://doi. org/10.1016/0021-9517(86)90183-1.

Sun Y, Zhang Z, Wong C. Study on mono-dispersed nano-size silica by surface modification for underfill applications. J Colloid Interface Sci. 2005;292(2):436-44. https://doi.org/10.1016/j. jcis.2005.05.067.

Wang X, Wu G, Guan N, Li L. Supported Pd catalysts for solvent-free benzyl alcohol selective oxidation: effects of calcination pretreatments and reconstruction of Pd sites. Appl Catal B. 2012;115:715. https://doi.org/10.1016/j.apcatb.2011.12.011. 
Whiting GT, Kondrat SA, Hammond C, Dimitratos N, He Q, Morgan DJ, et al. Methyl formate formation from methanol oxidation using supported gold-palladium nanoparticles. ACS Catal. 2014;5(2):637-44. https://doi.org/10.1021/cs501728r.

Wojcieszak R, Gaigneaux EM, Ruiz P. Direct methyl formate formation from methanol over supported palladium nanoparticles at low temperature. ChemCatChem. 2013a;5(1):339-48. https://doi. org/10.1002/cctc. 201200325 .

Wojcieszak R, Mateos-Blanco R, Hauwaert D, RG Carrazan S, M Gaigneaux E, Ruiz P. Influence of the preparation method on catalytic properties of $\mathrm{Pd} / \mathrm{TiO}_{2}$ catalysts in the reaction of partial oxidation of methanol. Curr Catal. 2013b;2(1):27-34. https://doi. org/10.2174/2211544711302010006.

Wojcieszak R, Ghazzal M, Gaigneaux EM, Ruiz P. Low temperature oxidation of methanol to methyl formate over Pd nanoparticles supported on $\gamma-\mathrm{Fe}_{2} \mathrm{O}_{3}$. Catal Sci Technol. 2014a;4(3):738-45. https://doi.org/10.1039/C3CY00859B.

Wojcieszak R, Karelovic A, Gaigneaux EM, Ruiz P. Oxidation of methanol to methyl formate over supported Pd nanoparticles: insights into the reaction mechanism at low temperature. Catal Sci Technol. 2014b;4(9):3298-305. https://doi.org/10.1039/C4CY00531G.

Xu J, Shang J-K, Chen Y, Wang Y, Li Y-X. Palladium nanoparticles supported on mesoporous carbon nitride for efficiently selective oxidation of benzyl alcohol with molecular oxygen. Appl Catal A. 2017;542:380-8. https://doi.org/10.1016/j.apcata.2017.05.036.

Yang X, Yan D. Long-afterglow metal-organic frameworks: reversible guest-induced phosphorescence tunability. Chem Sci. 2016;7(7):4519-26. https://doi.org/10.1039/c6sc00563b.
Yang X, Yan D. Direct white-light-emitting and near-infrared phosphorescence of zeolitic imidazolate framework-8. Chem Commun. 2017;53(11):1801-4. https://doi.org/10.1039/c6cc09706e.

Yang X, Lin X, Zhao Y, Zhao YS, Yan D. Lanthanide metal-organic framework microrods: colored optical waveguides and chiral polarized emission. Angew Chem. 2017;129(27):7961-5. https ://doi.org/10.1002/ange.201703917.

Yin Z, Zheng H, Ma D, Bao X. Porous palladium nanoflowers that have enhanced methanol electro-oxidation activity. J Phys Chem C. 2008;113(3):1001-5. https://doi.org/10.1021/jp807456j.

Yuan Q, Wu Z, Jin Y, Xu L, Xiong F, Ma Y, et al. Photocatalytic cross-coupling of methanol and formaldehyde on a rutile $\mathrm{TiO}_{2}$ (110) surface. J Am Chem Soc. 2013;135(13):5212-9. https:// doi.org/10.1021/ja400978r.

Zhang Q, Xu J, Yan D, Li S, Lu J, Cao X, et al. The in situ shapecontrolled synthesis and structure-activity relationship of Pd nanocrystal catalysts supported on layered double hydroxide. Catal Sci Technol. 2013;3(8):2016-24. https://doi.org/10.1039/ c3cy00143a.

Zhang Q, Li Y, Zhang L, Chen L, Liu Y, Lu Y. Structured nanoporousgold/Al-fiber: galvanic deposition preparation and reactivity for the oxidative coupling of methanol to methyl formate. Green Chem. 2014;16(6):2992-6. https://doi.org/10.1039/C3GC42561D

Zhao J, Shao M, Yan D, Zhang S, Lu Z, Li Z, et al. A hierarchical heterostructure based on Pd nanoparticles/layered double hydroxide nanowalls for enhanced ethanol electrooxidation. J Mater Chem A. 2013;1(19):5840-6. https://doi.org/10.1039/c3ta10588a. 